Running head: ATTENTIONAL BREADTH AND ATTACHMENT

Attentional breadth and proximity seeking in romantic attachment relationships Marieke Dewitte and Ernst H.W. Koster

Ghent University 


\begin{abstract}
The present study provides first evidence that attentional breadth responses can be influenced by proximity-distance goals in adult attachment relationships. In a sample of young couples, we measured attachment differences in the breadth of attentional focus in response to attachment-related cues. Results showed that priming with a negative attachment scenario broadens attention when confronted with pictures of the attachment figure in highly avoidant men. In women, we found that attachment anxiety was associated with a more narrow attentional focus on the attachment figure, yet only at an early stage of information processing. We also found that women showed a broader attentional focus around the attachment figure when their partner was more avoidantly attached. This pattern of results reflects the underlying action of attachment strategies and provides insight into the complex and dynamic influence of attachment on attentional processing in a dyadic context.
\end{abstract}

Key words: Adult Attachment, Attentional Breadth, Attentional Bias, Proximity Seeking, Individual Differences 
Attachment theory puts great emphasis on the role of attention in the etiology and maintenance of characteristic relationship behavior that forms the basis of one's attachment orientation (Bowlby, 1968/1982). According to the theory, attachment schemas determine whether attentional resources are directed towards or away from attachment-relevant cues, biasing further information processing in a goal-relevant and expectation-consistent manner (see Collins, Guichard, Ford, \& Feeney, 2004). One attentional component that has been fairly neglected in attachment research is attentional breadth. This is unfortunate because narrowing or broadening the attentional scope likely determines the processing of contextual cues and the (in)accurate decoding of attachment-figure signals (Derryberry \& Tucker, 1994). Given that attentional breadth likely influences cognitive processing of attachment cues, it may serve important functions for regulating proximity seeking in romantic relationships. The present study is the first to investigate attachment differences in the breadth of attentional focus when presented with attachment-figure related cues within couples.

\section{Attention in the Context of Adult Attachment}

The Attachment Behavioural System. A central idea in attachment theory is that attachment functioning is driven by an emotion regulation-system that is activated when feeling threatened, motivating people to obtain security by seeking proximity towards the attachment figure (Mikulincer \& Shaver, 2003). Individual differences exist in the regulatory strategies one can adopt to obtain this goal and these differences are determined by the (perceived) availability of the attachment figure. Typically, security will be attained through seeking proximity towards the attachment figure. However, when the attachment figure is perceived as being unavailable and proximity seeking efforts repeatedly failed in obtaining security, people are likely to adopt alternative strategies to regulate distress. These strategies map onto the dimensions of anxiety and avoidance which are assumed to underlie individual differences in attachment orientation (Brennan, Clark, \& Shaver, 1998). Hyperactivating 
strategies correspond with attachment anxiety, and would be characterized by exaggerated threat appraisals and vigilance to threat, which increase attentional focus and proximity seeking towards the attachment figure. Deactivating strategies, on the other hand, are typical for avoidant individuals. They aim at downregulating the attachment system by inhibiting emotional states and avoiding attention and proximity to the attachment figure as a means to maintain autonomy and interpersonal distance (for a review, see Mikulincer \& Shaver, 2003, 2007).

Attention and Attachment System Functioning. This model proposes that attachment strategies encompass different cognitive and behavioural processes that are driven by proximity and distance goals directed towards the attachment figure. One of the cognitive processes that has the potential to shape emotional distress reactions and support efficient goal-pursuit is attention. Attention is known to bias early processing of emotionally significant information and as such influences subsequent cognitive, emotional, and behavioural processes (e.g., Mathews, 1990). In the context of attachment, attention may serve two important functions in regulating attachment affect and behavior: (1) The triggering of the attachment system by directing attention to emotional and threatening information and (2) the regulation of proximity by directing attention to attachment-figure related cues.

Attention not only serves different functions, it also includes a variety of processes. According to Derryberry and Tucker (1994), emotional responses are modulated by two separate attentional processes, namely attentional orienting and attentional breadth. Other researchers have also identified attentional inhibition as an important ingredient of efficient emotion regulation (e.g., Zacks \& Hasher, 1994). Different attentional components have different functions (Weierich, Treat, \& Hollingworth, 2008). Whereas attentional orienting is important for selecting information that requires detailed processing, attentional inhibition plays a key role in preventing that goal-irrelevant information will be encoded and stored in 
memory. Attentional breadth, on the other hand, has a monitoring function and influences which and how much information will be processed simultaneously. As such, attentional tuning influences the breadth of activation of stored mental representations in long-term memory. It also assists in successful goal-pursuit because narrowing attention facilitates processing of goal-relevant cues, whereas broadening attention facilitates detection of potential means to attain these goals (Derryberry \& Tucker, 1994; Friedman \& Förster, 2010).

Attention as a Function of Threat Regulation. So far, only attentional orienting and attentional inhibition have been studied in the context of attachment. At the level of attentional orienting, studies have found that attachment anxiety and avoidance yield similar response patterns, namely attentional avoidance of attachment threat, which contradicts theoretical predictions (Dewitte, De Houwer, Koster, \& Buysse, 2007; Dewitte \& De Houwer, 2008). At the level of attentional inhibition, it was found that only attachment avoidance was associated with a stronger inhibition of negative affective stimuli. Attachment anxiety showed no relation with inhibitory responding to negative stimuli (Dewitte, 2011). Hence, in the case of attachment avoidance, the results on attentional orienting and inhibition were compatible, suggesting that both processes may be involved in the avoidant style of emotion regulation. Both biases may enable avoidant individuals to reduce the emotional impact of a triggering stimulus and limit further processing of unwanted negative affect (Fraley, Davis, \& Shaver, 1997; Fraley, Garner, \& Shaver, 2000). Note that there is also research showing that avoidant attentional biases are attenuated under cognitive load, suggesting that attentional inhibition of attachment information requires cognitive effort in avoidant individuals (Edelstein \& Gillath, 2008). In the case of attachment anxiety, however, results are less conclusive because there is yet no direct evidence of increased vigilance or impaired inhibition towards negative emotional information. 
Attention as a Function of Proximity Seeking. So far, we know little about the role of attention in regulating proximity once the attachment system has been activated by emotional cues. This is nevertheless important because an encounter with threat is known to activate proximity-related cognitions or mental representations of the attachment figure (see Mikulincer, Birnbaum, Woddis, \& Nachmias, 2000; Mikulincer, Gillath, \& Shaver, 2002). Such mental representations are likely to direct attentional resources towards attachment figure-related information. To test this hypothesis, previous research has examined selective attention to attachment-figure cues using a dot-probe task in which the name of the attachment figure, the own name, and the name of an acquaintance were presented in combination with a neutral name (Dewitte et al., 2007). It was shown that priming with attachment threat automatically activated an attentional bias towards the attachment name. This bias was consistently associated with attachment anxiety, whereas avoidance showed no association with selective attention for attachment cues. This is remarkable given that the aforementioned studies did indicate an important role of attentional processes in avoidant regulatory strategies (Diamond, Hicks, \& Otter-Henderson, 2006; Edelstein \& Gillath, 2008; Fraley \& Shaver, 1997; Fraley et al., 2000).

The Role of Attentional Breadth. To explore this issue further, more research is needed on the functional role of attention in directing proximity seeking responses. The study of attentional breadth may provide a useful perspective on this behalf. Given that attentional breadth can be moderated by emotional states, safety and danger cues, social stimuli, and motivational factors (Derryberry \& Reed, 2003; Gable \& Harmon-Jones, 2010), it is likely that attentional tuning is involved in regulating attachment responses. By broadening the attentional beam, people can monitor attachment threat and attachment figure-related cues in the environment, whereas narrowing the attentional focus may be functional to keep track of the attachment figure. It is thus plausible to assume that narrowing or broadening the 
attentional beam prepares the individual to engage in proximity seeking or distance keeping (respectively) in response to the attachment figure. Furthermore, by focusing more narrowly or broadly on attachment stimuli, attentional breadth is assumed to influence further processing at the conceptual and representational level. This means that attentional breadth influences the content and structure of attachment schemas, which has important implications for interpreting and responding to signals of the attachment figure and eventually for relational functioning.

A first test of attentional breadth as a function of proximity seeking has been conducted within a developmental perspective (Bosmans, Braet, Koster, \& De Raedt, 2009). Children were presented with pictures of the mother and unfamiliar women and needed to respond to targets that were presented close or far from this picture. Results showed that attachment-related cognitions did indeed influence the breadth of attention towards the attachment figure. More specifically, it was found that less secure children had a more narrow attentional focus around their mother. This suggests that insecure attachment representations influence cognitive processing aspects of proximity seeking. However, no differences were found in attentional responses as a function of the specific type of attachment insecurity.

\section{The Present Study}

The present study was designed to investigate the attention-proximity link in adult romantic relationships, using a similar task as the one used in the study by Bosmans and colleagues (2009). In a sample of couples, pictures of the partner and of unfamiliar persons were presented in the centre of the visual field, and a second stimulus was simultaneously presented at a random location around this central picture either close or far away from the picture. Greater difficulties to correctly identify the target when presented far from the centre compared with close to the centre, indicates that attention is narrowly focused on the central 
picture. To investigate the effect of attachment orientation on the time course of attentional responses, targets were presented at both short $(68 \mathrm{~ms})$ and longer $(250 \mathrm{~ms})$ presentation times. Because a presentation time of $68 \mathrm{~ms}$ is too brief to allow any saccades, this would reflect automatic narrowing of attention. Longer presentation times would indicate more elaborate and strategic attentional processing. Differentiating between automatic and strategic influences on attentional narrowing allows determining the amount of cognitive control that can be exerted on attentive processing. This is important because previous research did suggest that anxious and avoidant strategies may operate in different ways at different levels of processing (Diamond et al., 2006; Fraley et al., 2000; Mikulincer et al., 2000; Mikulincer et al., 2002).

Importantly, attentional breadth responses were measured in couples, allowing us to investigate actor and partner effects of attachment orientation on attention (Kenny, Kash, \& Cook, 2006). Given that the pursuit of proximity goals depends in part on the availability of the partner, it is relevant to investigate the impact of both attachment orientations on one's attentional responses. Previous research has indeed shown that individuals with insecurely attached partners display increased distress and proximity seeking (Dewitte, De Houwer, Goubert, \& Buysse, 2010). It is, however, not yet demonstrated whether such dyadic effects also exist at the attentional level.

We also induced a threat prime before the attentional breadth task. To investigate attentional breadth in the service of proximity seeking, it is important to first activate the attachment system because only then coping actions are required (Mikulincer et al., 2000). Attentional breadth responses to distress were then compared with attentional responses in a neutral condition. Furthermore, the induction of attachment threat may allow better differentiating between the impact of anxiety and avoidance on the breadth of attentional focus. 
Hypotheses. We predict that attachment anxiety is driven by proximity goals and is therefore associated with a more narrow focus on the attachment figure. Attachment avoidance would broaden attention as a means to distance from the attachment figure, especially in a threat-context. Given that avoidant strategies would include both automatic and strategic processes (Fraley et al., 2000), we predict a broadening of attention in more avoidant individuals at both presentation times. No predictions were made on the influence of attachment anxiety on the time course of attention, because evidence on the automatic or strategic nature of anxious strategies is inconclusive. We also expect that partner's attachment orientation will moderate attentional breadth responses such that attachment avoidance in one partner will evoke a broadening of attention in the other partner as a means to keep a defensive distance. Having a secure partner is likely to narrow attention towards this partner.

\section{Method}

\section{Participants}

Forty-five young adult heterosexual dating couples (total of 90 individuals) participated in the study in return for a monetary reward of 15 euros. They could subscribe to a university website on which several experiments are displayed (i.e., experimetrix). Participants had to be involved in a romantic relationship with their partner for at least 1 year (to assure that we were dealing with 'developed' attachment relationships, see Fraley \& Davis, 1997) ${ }^{1}$. Most of the female participants were psychology students. The mean age of the participants was 22.7 years for the men (ranging from 18 to 28 years) and 20.8 years for the women (ranging from 18 to 24 years) and the average relationship duration was 1.6 years (ranging from 1 to 4 years).

\section{Materials}


The stimuli for the attentional breadth task consisted of pictures of the partner and of an unknown person. 5 pictures were taken of each couple member, focusing specifically on their face. Participants were asked to show a neutral face without showing their teeth to avoid salience effects. Next, 5 pictures were taken of 5 different men and women unfamiliar to the participants. We used different persons to minimize potential effects of resemblance to own partner, attractiveness, or other salient properties. For the unknown category, participants were presented with pictures of the men and women (opposite-sex pictures) that participated two sessions before them. The pictures were taken by the experimenter using a digital photo camera and were edited to a format of $4 \mathrm{~cm}$ high and $3 \mathrm{~cm}$ wide. The attentional breadth task was programmed using the INQUISIT Millisecond Software Package (Inquisit 2.01, 2005) and presented on a Pentium II computer with a 19-inch colour monitor and a refresh rate of $100 \mathrm{~Hz}$. Participants responded using the computer mouse.

Attachment style was measured using a Dutch translation of the Experiences in Close Relationships Scale-revised (ECR-R; Fraley, Waller, \& Brennan, 2000; ECR-R-NL; Buysse \& Dewitte, 2004) which has proven to be internally consistent and adequate in terms of construct validity. The Anxiety scale taps fear of abandonment and the desire for interpersonal merger. The Avoidance scale assesses discomfort with closeness and dependence. Participants responded on a 7-points likert scale. As recommended, we asked our participants to fill in the questionnaire while holding their partner in mind. In the current sample, Cronbach alphas were high for the Anxiety subscale, $\alpha=.92$, as well as for the Avoidance subscale, $\alpha=.84^{2}$. The mean attachment scores were $2.39(S D=.67)$ for attachment anxiety and $2.03(S D=.77)$ for attachment avoidance.

\section{Procedure}


Before entering the laboratory, pictures were taken of both the male and female partner. Participants were told that they would participate in an experiment on dyadic effects in face recognition and we therefore needed to take some pictures. Then, 22 couples (44 participants) received priming instructions in which they were asked to write for several minutes about a situation in which they felt their partner didn't really love them. This task was presented as a visualisation exercise. The participants in the neutral condition ( 23 couples, 46 participants) waited an equal amount of time (compared to the priming condition) while the experimenter prepared the computer task. Participants were instructed not to communicate meanwhile. Both couple members were tested simultaneously while sitting averted from each other to avoid distraction. Participants were assigned to the negative and neutral condition in an alternating manner to avoid systematic differences between conditions. Note that both members of a couple received the same priming instructions. To measure the amount of distress elicited by the negative prime scenario, 12 visual analogue scales were administered before and after the prime. Participants were asked to place a mark on a 10-cm line according to how much distress, despair, loneliness, anxiety, sadness, insecurity, frustration, happiness, self-confidence, joy, satisfaction, and enthusiasm they experienced at that moment. We used continuous line rating scales to make it less likely that participants would remember their responses.

After the priming task, participants completed the attentional breadth task. For this task, they were seated in front of the computer, at a distance of exactly $27 \mathrm{~cm}$ from the screen using a chin rest to ensure accurate positioning. They were instructed to focus on the centre of the screen throughout the experiment and to use the chinrest to control the viewing distance. The attentional breadth task consisted of an instruction screen, 10 practice trials, and 128 test trials. Each trial started with the presentation of a central frame on a black background, surrounded by 16 grey dots with a diameter of $2 \mathrm{~cm}$ located at $4.5 \mathrm{~cm}$ (close trials at $10^{\circ}$ of 
the visual angle) and at $11.2 \mathrm{~cm}$ from the central frame (far trials at $25^{\circ}$ ). The grey dots were arranged in pairs of two (one close and one far dot, situated on one of eight imperceptible axes that came together in the central frame). After $400 \mathrm{~ms}$, a picture of the partner or neutral person was presented for 68 or $250 \mathrm{~ms}$ (depending on the presentation time), replacing the central frame. Together with the central picture, a small black circle with a diameter of $1.3 \mathrm{~cm}$ appeared either in one of the close or in one of the far dots. This black circle was the target stimulus that participants had to identify. Next, the face and the grey dots were masked for 20 ms to prevent impaired target detection by after-effects of the picture and target frames. The intertrial interval was 1000 ms (see Figure 1 for an overview).

After each trial, participants were presented with the question which picture they had seen (partner or neutral person). Responses on this question indicate whether participants were looking at the centre of the screen. Then, a second screen appeared with the question on which of eight axes the target was located. Participants were presented with 16 practice trials to get familiar with the procedure. The test phase consisted of 128 trials that were randomly presented in two blocks of 64 trials each, separated by a short break. In one block, picture and target were presented for $68 \mathrm{~ms}$. In the following block, the presentation time of target and picture was $250 \mathrm{~ms}$. Because we were primarily interested in individual differences, the presentation time was not counterbalanced across blocks to minimize error variance. In total, 8 categories were created with two picture types (partner versus neutral), two presentation times (68 and 250ms), and two distances (target presented close or far from the central picture). After the attentional breadth task, participants completed the ECR.

\section{Data Analytic Strategy}

Overall Analyses. For all analyses, we used only trials in which the picture was correctly identified, which ensures that attention was focused on the central picture. The latter 
is important because we want to measure whether attentional responses differ as a function of target identity (attachment figure versus neutral picture). This requires participants to focus on the central picture in order to differentiate between both trial types. Accordingly, all trials in which the central picture was incorrectly identified were deleted: $4.1 \%$ of the trials at $68 \mathrm{~ms}$ and $1.6 \%$ at $250 \mathrm{~ms}$.

The amount of correctly identified peripheral targets served as the main dependent variable. Performance on the attentional breadth task was examined by conducting a 2 (prime) X 2 (gender) X 2 (picture type) X 2 (presentation time) X 2 (distance) repeated measures ANOVA on the accuracy rates. Because dyadic data are interdependent and can thus not be treated as individual data points, gender was included as a within-couples factor (Kenny, 1996). Overall, more errors are expected at longer distance and faster presentation times.

Attachment Differences in Attentional Breadth. To address the research questions on attachment differences, we conducted a series of follow-up analyses, using Hierarchical Linear Modelling (HLM 6.06, Raudenbush \& Bryk, 2008). Given the dependency of dyadic data, the couple was the unit of analysis with female and male attentional responses nested within the couple. Within each couple, male and female responses are modelled separately (within the dyadic unit). To analyze the relation between prime condition, attachment dimensions, and attentional breadth, individual responses (nested within the couple) were modelled at level 1, and predictors were added at level 2 to explain the variability in the level 1 parameters. The predictors were prime condition, attachment anxiety, attachment avoidance, and the interaction between prime condition and the attachment scores (both two- and threeway interactions), for both couple members. The predictor variables were centred to avoid possible problems of multicollinearity. 


\section{Distress Induction}

First, we compared the amount of negative and positive feelings (means of the negative and positive VAS scales respectively) reported before and after the visualisation exercise. After the imagination of the attachment figure not loving them, participants generally reported more negative feelings, $t(42)=-4.40, p<.01$, and less positive feelings $t(42)=4.12, p<.01$, than before the negative prime. This indicates that our threat-prime was successful in eliciting distress. To examine the relation between attachment and the amount of distress elicited by the prime, we calculated difference-scores between the amount of positive and negative feelings reported before and after the threat-prime. A significant relationship was found between attachment anxiety and the amount of negative feelings, indicating that more anxiously attached individuals reported more negative feelings in response to attachment threat, $r=.38, p<.05$. Attachment avoidance, on the other hand, showed no significant relationship with the difference-scores of positive and negative feelings, $r$ 's $<.07$, $n s$; neither did they report a difference in mood before and after the threat-prime, $t<1.2, n s$.

\section{Overall Analyses}

A 2 (prime) X 2 (gender) X 2 (picture type) X 2 (presentation time) X 2 (distance) Repeated Measures ANOVA on the proportion of correctly identified targets yielded the predicted main effect of picture type, $F(1,44)=5.72, p<.05$, presentation time, $F(1,44)=$ $142.78, p<.001$, and of distance, $F(1,44)=344.25, p<.001$. Overall, participants responded more accurately to attachment pictures that were presented at close distance for $250 \mathrm{~ms}$. These main effects were qualified by a series of significant interaction effects. We found a significant interaction effect between presentation time and distance, $F(1,44)=39.63, p<$ .001. Also the Prime $\mathrm{X}$ Presentation Time $\mathrm{X}$ Distance interaction effect almost reached significance, $F(1,44)=3.58, p=.06 .^{3}$ Overall, participants yielded the most correct answers 
when targets were presented for $250 \mathrm{~ms}$ at short distances in the neutral prime condition and the least correct answers at targets presented for $68 \mathrm{~ms}$ at far distances in the negative prime condition. Finally, we found a significant Gender X Distance X Presentation Time interaction effect, $F(1,44)=11.46, p<.05$, showing that women made less errors at close trials presented for $250 \mathrm{~ms}$ in the negative prime condition. None of the other effects reached significance, all $F$ 's $<3.05$ (means and standard deviations are reported in Table 1). ${ }^{4}$

To interpret significant interaction effects and to present the results on individual differences in a more parsimonious way, we calculated difference scores. First, we calculated an attentional narrowing index (ANI) by subtracting the amount of correct responses at far trials from correct responses at close trials, both for the attachment figure and the neutral person. Then, we calculated a double difference score by subtracting the ANI score for neutral persons from the ANI score for the attachment figure because we are primarily interested in measuring differences in responding to the attachment figure relative to the neutral person. A higher ANI difference score $(\triangle \mathrm{ANI})$ implies a stronger attentional narrowing regarding pictures of the attachment figure. A lower ANI difference score indicates an attentional broadening effect.

\section{Attentional Breadth and Attachment Style}

A series of HLM models were conducted to test whether attachment anxiety and avoidance moderated attentional breadth responses. Because we are interested in the influence of attachment orientation on both automatic and strategic attentional processing, we performed the analyses for each presentation time separately.

Attentional Breadth Responses at $68 \mathrm{~ms}$. Adding prime condition and own attachment scores as predictor variables, and $\Delta \mathrm{ANI}$ as dependent variable, the interaction between prime condition and attachment avoidance yielded a significant effect, $\beta=3.72, p<.05$. This 
indicates that more avoidantly attached men showed a less narrow attentional focus on their partner when primed with a negative attachment scenario compared with a neutral condition. The interaction between attachment anxiety and avoidance also reached significance, $\beta=$ 2.20, $p<.05$, and was further qualified by a significant interaction effect between Prime $\mathrm{X}$ Anxiety X Avoidance, $\beta=-2.40, p<.05$. None of the other main and interaction effects were significant, all $p$ 's $>.10$. To interpret the interaction term, we examined $\Delta \mathrm{ANI}_{68 \mathrm{~ms}}$ scores, plotted at low $\left(25^{\text {th }}\right.$ percentile $)$ and high $\left(75^{\text {th }}\right.$ percentile $)$ values of male anxiety and avoidance in the threat and non-threat condition. This analysis indicated that particularly high avoidantlow anxious (i.e., dismissive) men showed a less narrow attentional focus in the negative prime condition, $\beta=-.87, t(42)=-2.53, p<.05$ (Fig. 2). Low anxious - low avoidant (i.e., secure) men, in contrast, were more narrowly focused on their attachment figure when primed with attachment threat, $\beta=.57, t(42)=1.70, p<.05$. The high avoidance-high anxiety slope and the anxiety-avoidance slopes in the non-threat condition were not significant, all $t$ 's < 1.44, $p$ 's >.10. In women, we found a marginally significant main effect of attachment anxiety, $\beta=1.39, p=.06$, indicating that higher scores on female anxiety were associated with a more narrow attentional focus on the partner. None of the other main and interaction effects were significant, all $p$ 's $>.10$.

Attentional Breadth Responses at $250 \mathrm{~ms}$. The analyses on the $\Delta \mathrm{ANI}_{250 \mathrm{~ms}}$ revealed a significant main effect of prime condition in men, $\beta=-.55, p<.01$, showing a general broadening of attention when primed with a negative attachment scenario compared with a neutral prime condition. In women, the effect of prime condition was not significant, $\beta=-.31$, $p>$.10. Analyses on male attentional responses also revealed a significant interaction effect between prime condition and attachment avoidance, $\beta=-.72, p<.01$. As presented in Figure 3, more avoidantly attached men showed a less narrow attentional focus in the negative prime condition compared to the neutral prime condition. In women, attachment avoidance yielded a 
significant main effect, $\beta=-.67, p<.01$, indicating that the more avoidance women reported, the less narrow their attention was focused on their partner. None of the other main and interaction effects were significant, all $p ’ s>.10$.

Partner-effects. The Partner X Attachment model revealed only one significant partner-effect. The interaction between male attachment anxiety and avoidance yielded a significant effect on the $\Delta \mathrm{ANI}_{250 \mathrm{~ms}}$ in women, $\beta=.26, p<.05$. Plotting these responses at low $\left(25^{\text {th }}\right.$ percentile $)$ and high $\left(75^{\text {th }}\right.$ percentile $)$ values of male anxiety and avoidance indicated that only women with high avoidant-low anxious (i.e., dismissive) partners showed a less narrow attentional focus on their partner, $\beta=-.34, t(42)=-2.30, p<.05$ (see Figure 4). The high anxiety and low avoidance slopes were not significant, all $t$ 's $<1.49, p$ 's $>.10 .^{5}$

\section{Discussion}

The present study aimed at investigating the role of attentional breadth in regulating proximity seeking as a function of attachment orientation and threat induction. In a sample of couples, we measured attachment differences in the breadth of attentional focus in response to attachment figure-cues compared to neutral cues. Consistent with theoretical expectations, we found that male attachment avoidance was associated with a broader attentional field around the attachment figure when primed with a relationship threat, both at short and long stimulus presentations. Secure men, on the other hand, narrowed their attentional focus around the attachment figure when feeling threatened, which fits with the primary attachment strategy of proximity seeking. The broadening effect in avoidant individuals can be interpreted as an attentional form of passively avoiding (i.e., not approaching) the attachment figure, which is likely driven by fear of intimacy and need for independence (Fraley \& Shaver, 1998; Gillath et al., 2006; Dewitte et al., 2008; Mikulincer \& Shaver, 2003, 2007; Simpson et al., 1992). Importantly, avoidant individuals broadened their attentional scope particularly when primed 
with a negative attachment context. This is congruent with research showing that avoidant strategies are activated mainly in the context of distress because only then coping actions are required (Fraley \& Shaver, 1998; Simpson et al., 1992). Such broad attentional focus may be functional to seek alternative sources of security and may as such assist in mood repair. It is also possible that the deactivating strategies of avoidant individuals have exerted their influence already at the onset of the regulation process by influencing the appraisal of the threat cue (Fraley \& Shaver, 1997; Fraley et al., 2000). Research has shown that avoidant individuals cognitively disengage from distress, allowing them to deny the importance of the threatening event. Placing less value at relational threat may thus reflect a functional strategy to prevent activation of attachment needs. Linking this with recent findings that affect of low motivational intensity broadens cognitive processing (Gable \& Harmon-Jones, 2010), it is plausible to suggest that avoidant individuals' broad attentional scope results from a lack of motivational interest when thinking about their attachment figure not loving them. Our mood measures may provide some support for this because no relationship was found between attachment avoidance and distress responses, suggesting that more avoidant individuals were emotionally unaffected by our distress induction.

Our study provides first evidence that this distancing effect also operates at the attentional level, suggesting that attentional breadth may be an important cognitive component of the avoidant approach to emotion regulation. The fact that similar results were found at short and long stimulus presentations attests to the robustness of this attentional broadening effect. It indicates that avoidant individuals broaden their attention to attachmentfigure cues even without having the opportunity and motivation to exert cognitive control. This fits with research showing that the defensive nature of avoidant strategies is reflected both at automatic and more strategic levels of responding (Fraley et al., 2000). Being able to cognitively disengage from attachment cues both at early and later stages of the regulatory 
process enables avoidant individuals to be effective in downregulating attachment system activation. This avoidant strategy may be reinforcing in the short run, but it is often maladaptive in the long run, because it has negative consequences for social interactions and eventually maintains insecurity. That is, broadening attention influences further processing of attachment cues, thereby biasing working memory access, interpretation of interpersonal signals, and attachment schema-activation, which then feedbacks into attentional processing. These feedback loops may have interpersonal consequences such as dismissing signs of attachment figure availability, inhibition of others needs, lack of empathic accuracy and compassion, and dysfunctional communication patterns (Dewitte, 2011; Mikulincer, \& Shaver, 2005; Simpson, et al., 2011), which then confirms avoidant individuals' negative beliefs about social interactions. Via its influence on higher-order cognitive processes, attentional breadth may thus have a marked influence on dyadic functioning. Furthermore, given that attentional breadth is implicated in regulating affect and memory processing, it is likely that attentional narrowing and broadening in response to relevant cues provokes psychopathological states, particularly emotional disorders (Derryberry \& Tucker, 1994).

Although the results on attachment avoidance were fairly consistent, we did observe a rather anomalous result when plotting the interaction term between priming and attachment avoidance in the long presentation condition. When no threat was present, high avoidant individuals tended to narrow their attention around the attachment figure. This is difficult to interpret in terms of defensive strategies and is not in line with other research showing that avoidant individuals tend to seek less information about their partner, even in the absence of threat (Rholes et al., 2007). However, considering that attentional broadening is primarily an emotion regulation strategy, it may be that the attachment system, even in the case of avoidant individuals, remains quiescent in the absence of negative emotions because no defensive actions are needed, thereby relying on proximity seeking. It is thus plausible to assume that 
the regulatory function of attachment avoidance is primarily manifest in the context of stressful, particularly relationship-threatening, situations.

Although gender differences are not common in attachment research and were thus not expected theoretically (Schmitt et al., 2003), we did find a different pattern of results for men and women. Attentional broadening in response to attachment cues was most consistently found in men. This fits with the common view that men prefer autonomy over closeness during relational stress, causing them to withdraw from their partner (see escape-conditioning model; Gottman \& Levenson, 1988). Through early socialisation experiences, men have learned to be more instrumental and less relationship-focused, resulting in increased withdrawal responses when confronted with relational stress (Christensen \& Heavy, 1990). We did, however, find no indication of increased proximity seeking in women, which would be reflected in attentional narrowing towards the partner.

Overall, the results in women were less robust. Female anxiety was found to be associated with a more narrow attentional focus on the attachment figure, yet only at short stimulus presentations. Although we have to be cautious when interpreting this result, it does fit well with research showing that cognitive processing of anxious individuals is driven by their strong wish for proximity and intimacy (Collins \& Allard, 2001; Gillath et al., 2006; Mikulincer et al., 2000). This may result in a constant monitoring of their attachment figure. The fact that such attentional narrowing was found only at an automatic level of processing is congruent with other research showing that, only at the automatic level, anxious individuals' wish for proximity produces vigilant responses to the attachment figure and a preparedness to engage in proximity seeking (Dewitte et al., 2007; Dewitte et al., 2008). This is, however, not translated directly into overt behaviour because they fear rejection and have doubts about support availability, which may cause ambivalence and approach-avoidance conflicts (e.g., Fraley \& Shaver, 1998; Mikulincer et al., 2002; Mikulincer, Shaver, Bar-On, Ein-dor, 2010). 
Also note that the relation between attachment anxiety and attentional narrowing did not depend on the presence of threat. This fits with the theoretical prediction that anxious individuals are chronically preoccupied with attachment concerns (Mikulincer \& Florian, 1995; Mikulincer et al., 2000). Unfortunately, we did not include a mood measure in the neutral condition, which prevented us from testing an explanation in terms of chronic distress. Yet, we did find that the more anxious individuals experienced more negative emotions in response to the induction of relational threat. Similar to men, we also found an association between attachment avoidance and attentional broadening, but, in women, this occurred only at longer presentation times and independently of threat induction. This suggests that attentional broadening in avoidant women is a more strategic and generalized phenomenon, whereas avoidant men broaden their attention only in the context of distress and at both levels of responding. It is difficult to provide a straightforward theoretical interpretation for this pattern of results.

Another interesting finding is that women broaden their attention when their partner was more avoidantly attached. Such broadening effect may signal the negative impact of attachment insecurity on couple relationships. That is, the emotional detachment and distancing behaviour of dismissive men may cause women to cognitively withdraw from their partner. The fact that this effect was found only in women fits with the idea that women take greater responsibility for their relationship, place more emphasis on dyadic relationships, and are therefore more likely to be influenced by the reactions of their partner (Gardner, Gabriel, \& Hochschield, 2002; Kiecolt-Glaser \& Newton, 2001). When plotting the interaction term, it also appeared that women tend to narrow their attention when their partner was more securely attached. This fits with the basic idea of attachment theory, stating that people seek proximity towards someone who can provide a safe haven and a secure base. 
Importantly, this is the first study showing that attentional responses can be moderated by partner's attachment orientation. Although there was only one dyadic effect, we believe this study does indicate the usefulness of measuring both one's own and partner's characteristics when trying to understand response patterns in couple relationships. It suggests that the attachment orientation of one partner influences the way in which the other partner regulates his/her proximity needs towards this person. Furthermore, using a sample of couples has surplus value in itself because it not only allows conducting dyadic analyses, it also increases the ecological validity of attentional research by providing direct evidence on the role of attention in couple functioning. Note that the observed correlations may have been weakened by our small sample size and the limited range of attachment scores. Hence, more research is needed to further explore dyadic links in attentional responding, using a larger and more heterogeneous sample of couples.

\section{Limitations}

This is the first study investigating the role of attentional breadth in adult attachment relationships, revealing important information on the cognitive component of proximity seeking to the attachment figure. Because of its exploratory nature, we cannot regard the current data as conclusive, but we do believe that the present study makes a timely point concerning the role of attentional breadth in attachment research, which paves the way for further investigation. An important strength of this study is that we used a cognitive task that was adapted to the individual participant by using pictures of the "real" partner, which increases the ecological validity of the present research.

One potential weakness of the present study is that attentional breadth responses towards attachment-figure's pictures are measured relative to an unknown person's pictures. These pictures systematically differ from the attachment figure in terms of salience and 
familiarity, which means that certain features of the attachment figure could be more prominent compared to the neutral person. The latter may introduce some ambiguities for interpreting the present findings. Future research is thus needed to exclude such alternative explanations by using pictures of a known person with whom one is familiar, but who does not serve attachment functions (e.g., classmates, colleagues). Also, we did not include measures of likeability and attractiveness, which could differ between neutral and attachment persons. Note, however, that the observed relationships between the attentional breadth index and attachment styles do seem to suggest that our results are driven by attachment processes and not salience or likeability effects. Furthermore, the fact that our results were compatible with attachment theory and other research on attentional processing validates the present research design.

A related methodological remark concerns the fact that we used a double difference score to examine the relation between attachment style and attentional breadth. The most important reason to calculate difference scores is that we were not interested in the relation between attachment orientation and the absolute ANI scores for the attachment and neutral person. We primarily wanted to measure whether attentional responses differ when confronted with the attachment figure compared with a neutral person as a function of prime condition and attachment orientation. Although such difference-scores are consistent with our theorizing and useful to interpret individual differences, it is unclear whether the observed effects are driven by the attachment figure, the neutral person or both. Note, however, that the omnibus analyses did indicate that the overall effects are driven by both trial types. This suggests that pictures of the neutral person evoked similar attentional effects as the attachment figure. We should take this into account when interpreting the effects on attachment differences. 
Another possible shortcoming is that the negative prime and control conditions were not directly comparable because in the neutral condition participants were not given any particular instructions. Furthermore, we did not administer a mood measure in the neutral condition. Accordingly, we cannot verify whether any findings related to condition are due to negative affect, thinking about the partner, or just thinking about something. However, because our findings are congruent with theoretical predictions and previous studies on proximity seeking - in which the neutral condition did include a mood measure and a prime instruction (e.g., describing the route to university) (Dewitte et al., 2008) - we do believe that our findings can be explained in terms of the stress-attachment link.

Finally, we did not include measures of relational quality or perceived partner responsiveness, which could mediate the effect of distress on attentional components of proximity seeking in couples. The inclusion of such measures would also be useful to rule out the possibility that there were differences in relationship satisfaction between the experimental conditions, which could have influenced the results.

\section{Conclusion}

The present study provided preliminary data on the role of attentional breadth for regulating proximity seeking towards the attachment figure as a function of anxious and avoidant attachment strategies. It is also the first study investigating attentional processing in a sample of couples, which is important to better understand the dynamics of proximity seeking within attachment relationships. Because attachment theory puts great emphasis on attentional processes for developing and maintaining attachment insecurity, further experimental research into the functional role of attention is much needed to advance our knowledge on the source of emotional dysregulation in insecure individuals. 


\section{Footnotes}

${ }^{1}$ To assure that everyone considered their partner as primary attachment figure, we presented participants with the WHOTO scale before completing the experiment. This scale consists of six questions referring to the three critical features that distinguish attachment figures from non-attachment figures (proximity seeking and separation distress, safe haven, and secure base; Hazan \& Zeifman, 1994). For each question, participants had to write the name of the person that best served each of these functions. The person who was listed most frequently was labeled as the primary attachment figure. In case of an ex aequo, we asked the participants to chose one of them as preferred attachment figure (see Fraley \& Davis, 1997). As expected, every participant labeled their partner as primary attachment figure.

${ }^{2}$ The two priming groups were similar with regard to their attachment scores, $t(88)=.12, p>$ .10 for attachment anxiety and $t(88)=.38, p>.10$ for attachment avoidance. In both priming groups, anxiety and avoidance scores were significantly correlated, $r=.58, p<.01$ in the neutral prime condition and $r=.55, p<.01$ in the negative prime condition.

${ }^{3}$ Note that these main and interaction effects justify the calculation of difference scores.

${ }^{4}$ The use of double difference scores might introduce some ambiguity in interpreting the results because it is unclear whether the observed relations between attachment style and attentional responses are driven by the attachment figure, the neutral person or both. To address this issue, we want to report that the repeated measures ANOVA revealed a significant three-way interaction between Prime X Picture Type X Distance, $F(1,85)=7.70$, $p<.01$. To interpret this significant interaction effect, a series of t-tests were conducted, using the attentional difference scores (close trials - far trials) as dependent variables. Results showed that, in both the neutral and negative prime condition, the ANI attachment scores did not significantly differ from the ANI neutral scores at both presentation times, $t<1.91, n s$. 
Although these results say nothing about individual differences, they do suggest that the overall attentional effects are driven by both attachment-figure and neutral-person trials.

${ }^{5}$ As relationships develop over time, partners' roles as attachment figures may have increasing importance, especially in the case of young adults. Therefore, we included relationship length as a moderator variable in our analyses, which did not affect the general pattern of results. Note that the range of relationship duration was quite limited (1 to 4 years), with most couples having a developing relationship. This might explain why relationship length had little effect on attentional breadth scores as a function of attachment. 


\section{References}

Bekkering, H., \& Neggers, S.F. (2002). Visual search is modulated by action intentions. Psychological Science, 19, 362-370.

Bosmans, G., Braet, C., Koster, E., \& De Raedt, R. (2009). Attachment security and attentional breadth towards the attachment figure in middle childhood. Journal of Clinical and Adolescent Psychology, 38, 872-882.

Bowlby, J. (1982/1969). Attachment and Loss: Vol.1. Attachment (2nd ed.) New York: Basic Books.

Brennan, K. A., Clark, C. L., \& Shaver, P. R. (1998). Self-report measurement of adult romantic attachment: An integrative overview. In J. A. Simpson \& W. S. Rholes (Eds.), Attachment theory and close relationships (pp.46-76). New York: The Guilford Press.

Christensen, A., \& Heavy, C. L. (1990). Gender and social structure in the demand/withdraw pattern of marital conflict. Journal of Personality and Social Psychology, 59, 73 - 81

Collins, N. L., \& Allard, L. M. (2001). Cognitive representations of attachment: the content and function of working models. In G. J. O. Fletcher \& M. S. Clark (Eds.), Blackwell Handbook of Social Psychology: Vol. 2. Interpersonal Processes (pp. 60-85). United Kingdom: Blackwell Publishers.

Collins, N. L., Guichard, A. C., Ford, M. B., Feeney, B. C. (2004). Working models of attachment: New developments and emerging themes. In W.S Rholes, \& J.A. Simpson, Adult attachment: Theory, research, and clinical implications (pp. 196-239). New York: Guilford Press. 
Derryberry, D., \& Reed, M.A. (2003). Anxiety-related attentional biases and their regulation by attentional control. Journal of Abnormal Psychology, 111, 225-236.

Derryberry, D., \& Reed, M.A. (1996). Regulatory processes and the development of cognitive representations. Development and Psychopathology, 8, 215-234.

Derryberry, D., \& Tucker, D.M. (1994). Motivating the focus of attention. In P.M. Niedenthal \& S. Kitayama (Eds.), The heart's eye: Emotional influences in perception and attention (pp. 167-196). San Diego, CA: Academic Press.

Dewitte, M. (2011). Adult Attachment and inhibition of interpersonal information. Cognition \& Emotion, 25, 612-625.

Dewitte, M., De Houwer, J., Koster, E.H.W., \& Buysse, A. (2007). What's in a name: Attachment-related attentional bias. Emotion, 7, 535-545.

Dewitte, M., \& De Houwer, J. (2008). Adult attachment and attention to positive and negative emotional face expressions. Journal of Research in Personality, 42, 498-505.

Dewitte, M., De Houwer, J., Buysse, A., \& Koster, E.H.W. (2008). Examining proximity seeking in adult attachment: The role of automatic approach-avoidance tendencies. British Journal of Social Psychology, 47, 557-573.

Dewitte, M., De Houwer, J., Goubert, L., \& Buysse, A. (2010). A multi-modal approach to the study of attachment-related distress. Biological Psychology, 85, 149-162.

Dewitte, M., Koster, E.H.W., De Houwer, J., \& Buysse, A. (2007). Attentive processing of threat and adult attachment: A dot-probe study. Behaviour Research and Therapy, 45, $1307-1317$. 
Diamond, L. M., Hicks, A. M., Otter-Henderson, K. A. (2006). Physiological evidence for repressive coping among avoidantly attached adults. Journal of Social and Personal Relationships, 23, 205-229.

Edelstein, R.S., \& Gillath, O. (2008). Avoiding interference: Adult attachment and emotional processing biases. Personality and Social Psychology Bulletin, 34, 171-181.

Eysenck, M.W. (1992). Anxiety: The Cognitive Perspective. Erlbaum: Hove.

Fraley, R. C., \& Davis, K. E. (1997). Attachment formation and transfer in young adult's close friendships and romantic relationships. Personal Relationships, 4, 131-144.

Fraley, R. C., Davis, K. E., \& Shaver, P. R. (1998). Dismissing-avoidance and the defensive organization of emotion, cognition, and behavior. In J. A. Simpson \& W. S. Rholes (Eds.), Attachment theory and close relationships (pp. 249-279). New York: Guilford Press.

Fraley, R. C., Garner, J. P., \& Shaver, P. R. (2000). Adult attachment and the defensive regulation of attention and memory: Examining the role of preemptive and postemptive defensive processes. Journal of Personality and Social Psychology, 79, 816-826.

Fraley, R. C., \& Shaver, P. R. (1997). Adult attachment and the suppression of unwanted thoughts. Journal of Personality and Social Psychology, 79, 816-826.

Fraley, R. C., \& Shaver, P. R. (1998). Airport separations: A naturalistic study of adult attachment dynamics in separating couples. Journal of Personality and Social Psychology, 75, 1198-1212. 
Fraley, R. C., Waller, N. G., \& Brennan, K. A. (2000). An item response theory analysis of self-report measures of adult attachment. Journal of Personality and Social Psychology, 78, 350-365.

Friedman, R. S. \& Förster, J. (2010). Implicit affective cues and attentional tuning: An integrative review. Psychological Bulletin, 136, 875-893.

Gardner, W. L., Gabriel, S., \& Hochschield, L. (2002). When you and I are "we" you are not threatening: The role of self-expansion in social comparison. Journal of Personality and Social Psychology, 82, 239-251.

Gillath O., Mikulincer, M., Fitzsimons, G. M., Shaver, P. R., Schachner, D. A., \& Bargh, J. A., (2006). Automatic activation of attachment-related goals. Personality and Social Psychology Bulletin, 32, 1375-1388.

Gable, P., \& Harmon-Jones, E. (2010). The motivational dimension of affect: Implications for breadth of attention, memory and cognitive categorization. Cognition \& Emotion, 24, 322-337.

Gottman, J. M., \& Levenson, R. W. (1988). The social psychophysiology of marriage. In P. Noller \& M. A. Fitzpatrick (Eds.), Perspectives on marital interaction (pp. 182-199). Clevedon, England: Multilingual Matters.

Kenny, D. A. (1996). Models of non-independence in dyadic research. Journal of Social and Personal Relationships, 13, 279-294.

Kenny, D. A., Kashy, D. A., \& Cook, W. L. (2006). Dyadic data analysis. The Guilford Press: New York Londen. 
Kiecolt-Glaser, J.K., \& Newton, T.L. (2001). Marriage and health: His and hers. Psychological Bulletin, 127, 472-503.

Lang, P.J. (1995). The emotion probe. American Psychologist, 50, 372-385.

Mathews, A. (1990). Why worry? The cognitive function of anxiety. Behavioral Research and Therapy, 28, 455-468.

Mikulincer, M., Birnbaum, G., Woddis, D., \& Nachmias, G. (2000). Stress and accessibility of proximity-related thoughts: Exploring the normative and intraindividual components of attachment theory. Journal of Personality and Social Psychology, 78, 509-523.

Mikulincer, M., Gillath, O., \& Shaver, P. R., (2002). Activation of the attachment system in adulthood: Threat-related primes increase the accessibility of mental representations of attachment figures. Journal of Personality and Social Psychology, 83, 881-895.

Mikulincer, M., \& Florian, V. (1995). Appraisal and coping with a real-life stressful situation: The contribution of attachment styles. Personality and Social Psychology Bulletin, 21, 406-414.

Mikulincer, M., \& Shaver, P. R. (2003). The attachment behavioural system in adulthood: Activation, psychodynamics, and interpersonal processes. Advances in Experimental Social Psychology, 35, 53-152.

Mikulincer, M., \& Shaver, P. R. (2004). Security-based self-representations in adulthood: Contents and processes. In W. S. Rholes \& J. A. Simpson (eds.), Adult attachment: Theory, research, and clinical implications (pp. 159-195). New York: Guilford Press. 
Mikulincer, M., \& Shaver, P. R. (2005). Attachment security, compassion, and altruism. Current Directions in Psychological Science, 14, 34-38.

Mikulincer, M., \& Shaver, P. R. (2007). Attachment in adulthood: Structure, dynamics, and change. New York: Guilford Press.

Mikulincer, M., Shaver, P.R., Bar-On, N., \& Ein-Dor, T. (2010). The pushes and pulls of close relationships: Attachment insecurities and relational ambivalence. Journal of Personality and Social Psychology, 98, 450-468.

Schmitt D.P., Alcalay L., Allensworth M., (...) \& Zupaneie, A. (2003). Are men universally more dismissing than women? Gender differences in romantic attachment across 62 cultural regions. Personal Relationships, 10, 307-331.

Simpson, J. A., Kim, J., Fillo, J., Ickes, W., Rholes, W. S., Oriña, M. M., \& Winterheld, H. A. (2011). Attachment and the management of empathic accuracy in relationshipthreatening situations. Personality and Social Psychology Bulletin, 37, 242-254.

Simpson, J. A., Rholes, W. S., \& Nelligan, J. S. (1992). Support seeking and support givings within couples in an anxiety-provoking situation: The role of attachment styles. Journal of Personality and Social Psychology, 62, 434-446.

Weierich, M.R., Treat, T.A., \& Hollingworth, A. (2008). Theories and measurement of visual attentional processing in anxiety. Cognition and Emotion, 22, 985-1018.

Zacks, R.T., \& Hasher, L. (1994). Directed ignoring: Inhibitory regulation of working memory. In D. Dagenbach \& T.H. Carr (Eds.), Inhibitory processes in attention, memory, and language (pp. 241-264). San Diego, CA: Academic Press. 
Table 1. Means and standard deviations of the proportion correctly identified targets per condition in the Attentional Breadth Task as a function of Gender

\begin{tabular}{|c|c|c|c|c|c|c|c|c|}
\hline & \multicolumn{4}{|c|}{ Neutral prime } & \multicolumn{4}{|c|}{ Negative prime } \\
\hline & \multicolumn{2}{|c|}{ Attachment figure } & \multicolumn{2}{|c|}{ Neutral person } & \multicolumn{2}{|c|}{ Attachment figure } & \multicolumn{2}{|c|}{ Neutral person } \\
\hline & close & far & close & far & close & far & close & far \\
\hline \multicolumn{9}{|c|}{ Male partners } \\
\hline $68 \mathrm{~ms}$ & $.90(.12)$ & $.50(.19)$ & $.89(.12)$ & $.47(.24)$ & $.78(.21)$ & $.47(.20)$ & $.79(.19)$ & $.45(.19)$ \\
\hline $250 \mathrm{~ms}$ & $.95(.08)$ & $.66(.18)$ & $.91(.10)$ & $.64(.20)$ & $.80(.22)$ & $.65(.22)$ & $.83(.21)$ & $.63(.23)$ \\
\hline \multicolumn{9}{|c|}{ Female partners } \\
\hline $68 \mathrm{~ms}$ & $.85(.20)$ & $.47(.21)$ & $.82(.19)$ & $.50(.20)$ & $.89(.09)$ & $.48(.23)$ & $.89(.10)$ & $.43(.23)$ \\
\hline $250 \mathrm{~ms}$ & $.92(.09)$ & $.60(.22)$ & $.88(.19)$ & $.58(.22)$ & $.94(.07)$ & $.64(.20)$ & $.97(.06)$ & $.67(.21)$ \\
\hline
\end{tabular}


Fig1. Stimulus presentation of the Attentional Breadth Task 


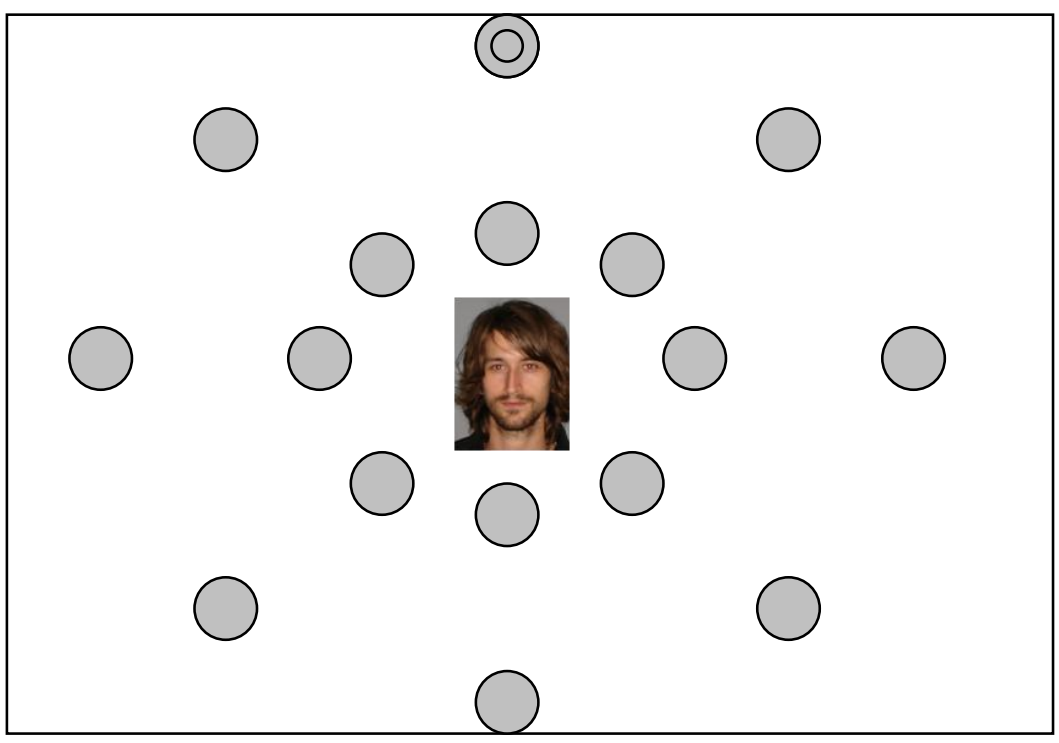

Screen 1 (far trial)

Who did you see in the middle of the screen? Select "1" or "2"

\section{Partner}

1

\section{Unfamiliar}

2

Screen 2

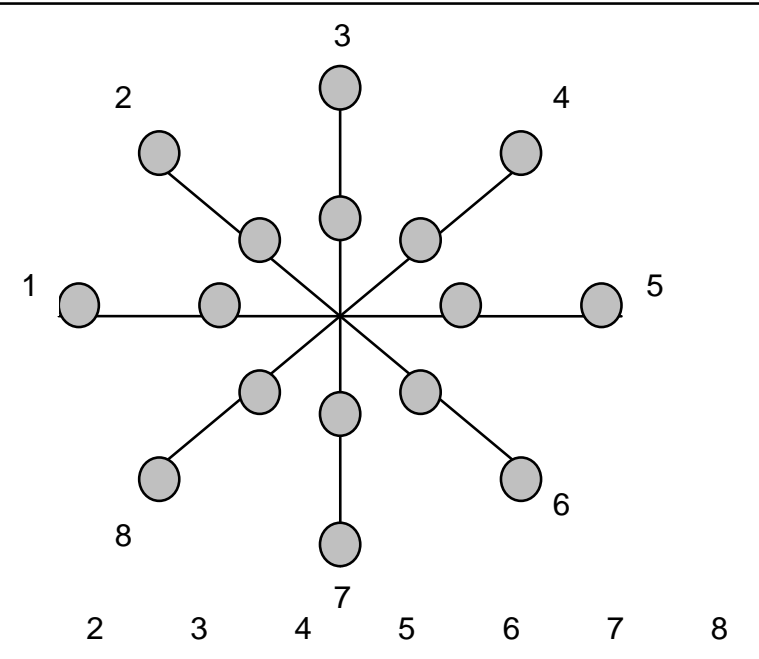

On which axis did the circle appear? Select the corresponding number. 
Fig 2. Male attentional breadth responses at $68 \mathrm{~ms}$ as a function of attachment avoidance and prime condition

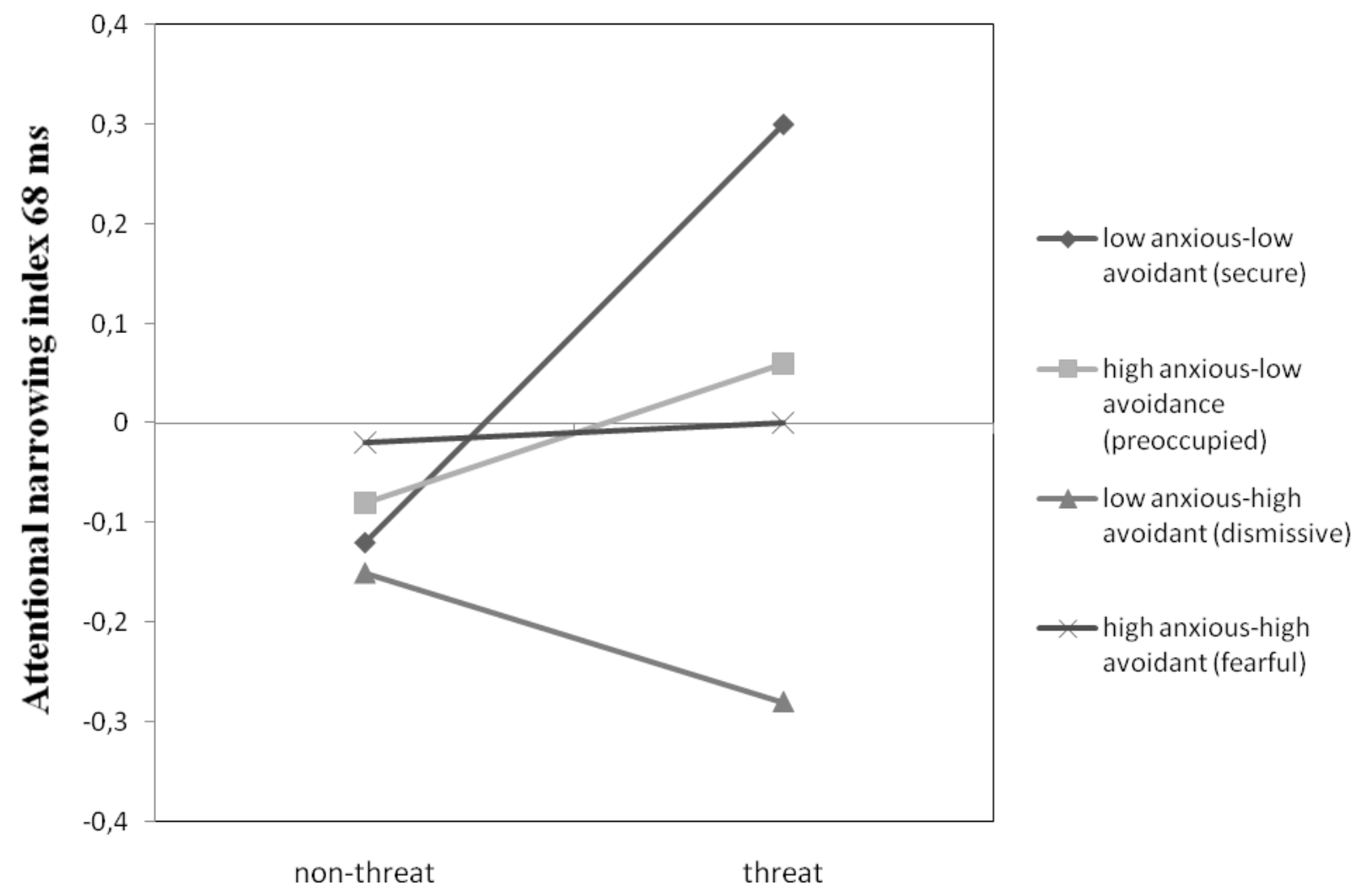

* Higher/positive values indicate attentional narrowing; Lower/negative scores indicate attentional broadening 
Fig. 3. Male attentional breadth responses at $250 \mathrm{~ms}$ as a function of attachment avoidance and prime condition

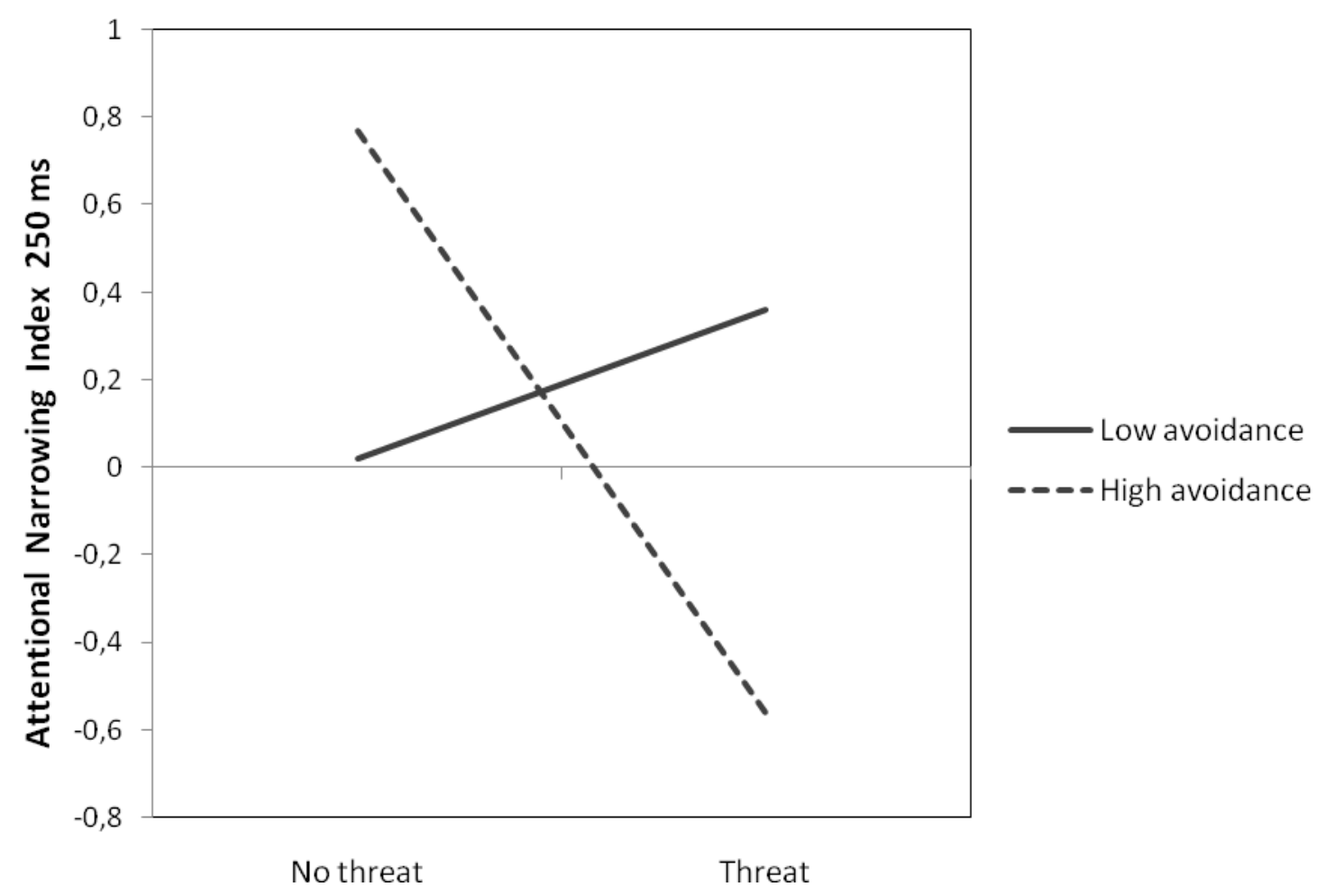

* Higher/positive values indicate attentional narrowing; Lower/negative scores indicate attentional broadening 
Fig 4. Attentional breadth responses in women plotted at low and high values of male anxiety and avoidance

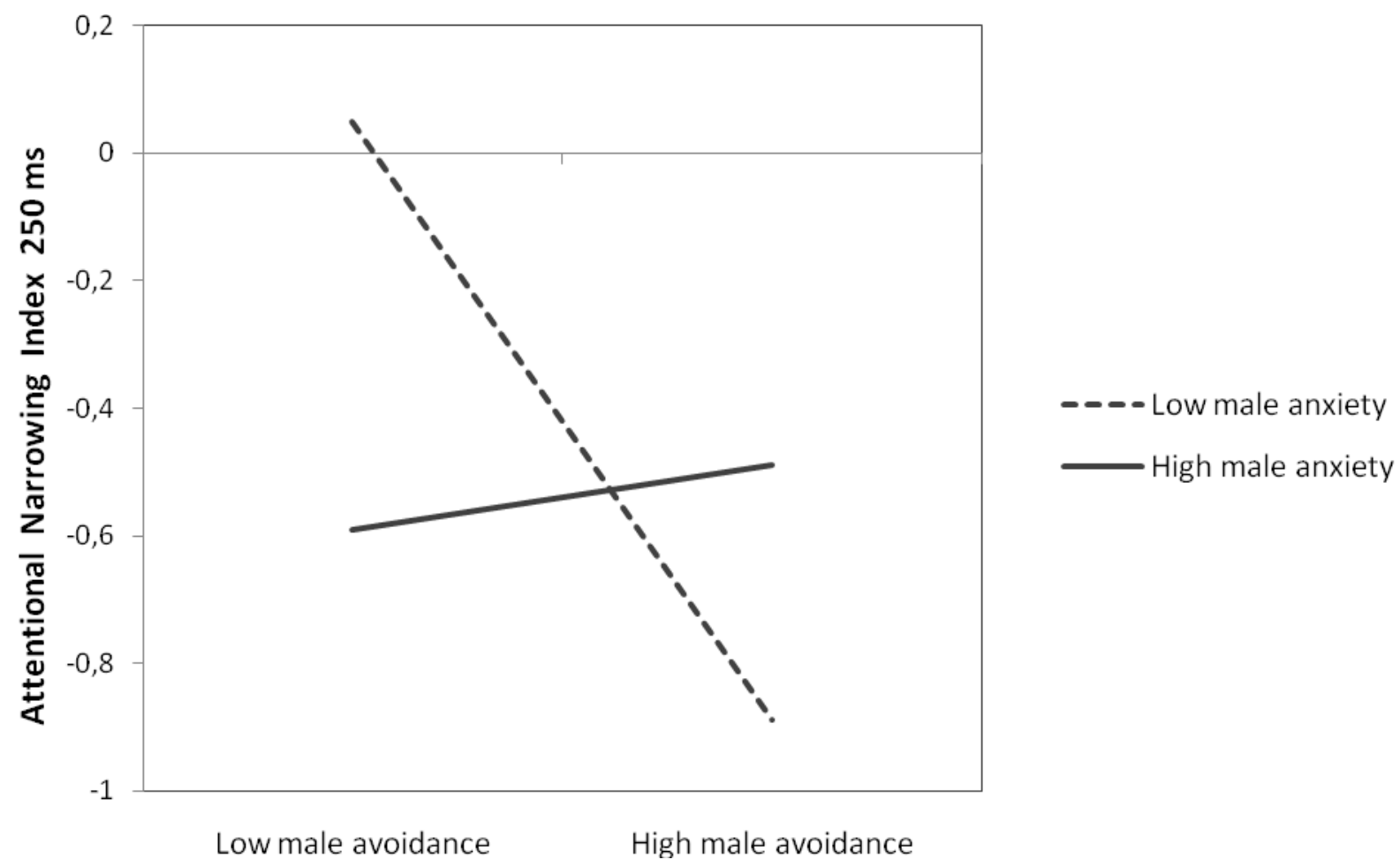

* Higher/positive values indicate attentional narrowing; Lower/negative scores indicate attentional broadening 